\title{
Los estudios del coste del alcoholismo: marco conceptual, limitaciones y resultados en España
}

\author{
García-Sempere, A.; Portella, E. \\ Antares Consulting
}

Enviar correspondencia a: Aníbal García-Sempere. Antares Consulting S.A. Vía Augusta, $2001^{\circ}$ sur. 08021 Barcelona. E-mail: agarcias@antares-consulting.com

\section{RESUMEN}

Los estudios sobre las consecuencias económicas del alcoholismo utilizan usualmente la metodología de "estudio del coste de la enfermedad", donde el impacto de un determinado problema de salud sobre el bienestar de la sociedad se valora mediante la cuantificación de los costes de los recursos empleados para su prevención y tratamiento, los costes legales atribuibles, las pérdidas de productividad derivadas de la morbilidad y mortalidad específicas y la pérdida de años de vida ajustados por calidad. En España, y siguiendo esta metodología, se han valorado los costes del alcoholismo en más de 630.000 millones de pesetas anuales. Estos estudios han recibido críticas en relación con la identificación, medida y cuantificación de los costes, y su utilidad es discutida. Sin embargo, y pese a determinadas limitaciones, los estudios del coste de la enfermedad aproximan a la magnitud del problema, contribuyen a identificar los aspectos del problema que suponen un coste más elevado y, ayudan a orientar las políticas. El ajuste a las guías metodológicas y la explicitación detallada de las fuentes y métodos utilizados es un requisito necesario para su credibilidad.

Palabras clave: economía, coste de la enfermedad, alcoholismo, salud pública.

\begin{abstract}
Studies on economic consequences of alcoholism usually use "cost-of-illness studies" methodology, where impact on society's well-being of a certain problem of health is valued by means of quantifying the costs of resources used for its prevention and treatment, the attributable legal costs, the productivity losses derived from specific morbidity and mortality and the loss of quality adjusted life years. In Spain, and following this methodology, costs of alcoholism have been valued in more than 630,000 annual million pesetas. These studies have received critics in relation to the identification, measurement and quantification of costs, and their utility is been discussed. Nevertheless, and in spite of certain limitations, cost-of-illness studies approximate to the magnitude of the problem, contribute to identify aspects of the problem that suppose a higher cost and help to orient policies. Adjustment to methodology guides and explicit sources and used methods are necessary requirements for its credibility.
\end{abstract}

Key words: cost of illness, economy, alcohol abuse, public health

\section{INTRODUCCIÓN}

consumo y sobreconsumo de alcohol conlleva una amplia serie de consecuencias negativas para la salud (ver tabla 1) y el bienestar de la sociedad que pueden ser identificadas, medidas y cuantificadas monetariamente en forma de costes del alcoholismo. La importancia de los costes sociales es la principal justificación para el desarrollo de polí- 
TABLA 1. Alteraciones relacionadas con el alcoholismo

ALT. COMPORTAMIENTOY PSICOSIS

Intoxicación aguda

Envenenamiento por alcohol

Amnesia

Dependencia del alcohol

Síndrome de abstinencia

Delirium tremens y Alucinosis aguda

Depresión

Intentos de suicidio / suicidio

\section{NEUROLÓGICAS}

Epilepsia

Neuropatías periféricas

Atrofia cerebral

Ataxia cerebelosa

Síndrome de Wernicke-Korsakoff

Traumatismos craneo-encefálicos

Accidentes cerebro-vasculares

\section{GASTROINTESTINALES}

Carcinoma orofaringeo

Esofagitis y alt. Agudas de esófago

Síndrome de Mallory-Weiss

Varices esofágicas

Carcinoma esofágica

Gastritis erosivas y atróficas

Carcinoma gástrico

Alt. Motilidad digestiva

Mala absorción intestinal

Carcinoma de colon

Disfunciones pancreáticas

Pancreatitis crónica

Carcinoma de páncreas

Degeneración grasa hepática

Hepatitis alcohólica

Cirrosis

Carcinoma hepatocelular

\section{CARDIOVASCULAR}

Arritmias cardiacas

Cardiomiopatía alcohólica

Beriberi

Hipertensión

Coronariopatías

\section{RESPIRATORIO}

Apnea de sueño

Enf. Pulmonar obstructiva crónica

Neumonía y absceso pulmonar

Tuberculosis pulmonar

Carcinoma de laringe

Cáncer de pulmón

\section{ENDOCRINOY METABOLISMO}

Hipo e hiperglicemia

Diabetes

Gota

Acidosis láctica

Alt. Del metabolismo de minerales

Deficiencias nutricionales

\section{REPRODUCTIVO}

Disminución función testicular

Disminución función ovárica

Carcinoma de mama

\section{MUSCULOESQUELETICO}

Miopatía aguda y crónica

Necrosis isquémica de la cabeza femoral

Osteoporosis

\section{HEMATOLOGÍA}

Anemia

Disminución respuesta a la infección

Trombocitopenia

\section{LESIONES TRAUMÁTICAS Y VIOLENCIA}

\section{INTERACCIONES CON FÁRMACOS}

\section{ALTERACIONES DEL EMBARAZOY PARTO}

Aborto espontáneo

Bajo peso al nacer y mortalidad perinatal

Retraso en el desarrollo

Anomalías congénitas

Síndrome fetal alcohólico

Pseudo-Cushing en neonatos

Síndrome de abstinencia en neonatos 
ticas públicas frente al alcoholismo (tanto en forma de regulaciones especiales y políticas fiscales, como de políticas -coercitivas o noespecíficas), y disponer de estimadores consistentes de estos costes se considera fundamental para conocer la relevancia del problema, priorizar las actuaciones públicas y, hasta cierto punto, evaluar programas y políticas.

El estudio de los costes económicos del consumo de alcohol se aborda usualmente mediante una metodología específica denominada estudios del coste de la enfermedad (CdE, cost of illness studies) $)^{1-5}$, donde el impacto de un determinado problema de salud sobre el bienestar de la sociedad es valorado mediante la cuantificación de los costes de los recursos empleados para su prevención y tratamiento, los costes legales atribuibles, las pérdidas de productividad derivadas de la morbilidad y mortalidad específicas, y la pérdida de años de vida ajustados por calidad (Quality Adjusted Life Years), respecto a un escenario ideal de inexistencia del problema ${ }^{4}$. Mas allá de Estados Unidos y Canadá, muy pocos países disponen de estudios de $\mathrm{CdE}$ alcohólica rigurosos ${ }^{6}$. Aun en estos casos, y pese a la importante estandarización de la metodología de los estudios de $\mathrm{CdE}^{1,4,7-11}$, los estimadores están sujetos a discusión por algunas de sus asunciones, insuficiencias de las fuentes de información, variaciones en los diseños y limitaciones metodológicas ${ }^{12-18}$ que, en conjunto, producen importantes diferencias en los resultados de las estimaciones ${ }^{5,6}$.

El objetivo de este trabajo es revisar el marco conceptual de los estudios de coste de la enfermedad, con especial referencia a los estudios de costes del consumo de alcohol, revisar los estudios españoles sobre el tema, y discutir las limitaciones de estos estudios, así como sus posibles aplicaciones.

\section{FUNDAMENTOS ECONÓMICOS DE LOS ESTUDIOS DEL COSTE DE LA ENFERME- DAD}

En su expresión mas simple, los estudios del $\mathrm{CdE}$ combinan datos epidemiológicos con datos económicos para obtener una cifra, en unidades monetarias, que informa de los costes que un determinado problema de salud impone a la sociedad ${ }^{4,19}$. Esta cifra ofrece una idea de la importancia económica y social del problema estudiado y usualmente, su magnitud es lo suficientemente importante para atraer la atención de la sociedad sobre el tema, en especial de aquellos que pueden tomar decisiones al respecto. Los primeros estudios del CdE se realizaron en la década de los 50 sobre accidentes de tráfico ${ }^{20}$ y enfermedades mentales ${ }^{21}$ y los primeros sobre costes del alcoholismo fueron realizados en Australia a finales de los años $60^{22}$ y en Estados Unidos a principios de los $70^{23,24}$. Pese a que la mayor parte de estos estudios se han realizado en Estados Unidos, en la última década se han desarrollado trabajos de interés en Australia ${ }^{3,25}$, Canada ${ }^{26-28}$, Nueva Zelanda ${ }^{29}$, Japón ${ }^{30}$, en los propios Estados Unidos $^{5,11}$ y también en España ${ }^{31-35}$, y se ha sugerido que con los avances teóricos y metodológicos ha aumentado la comparabilidad y consistencia entre estudios ${ }^{5}$.

Las diferentes aproximaciones de los estudios de CdE parten del concepto de "coste de oportunidad" presuponiendo que, de no existir la enfermedad (alternativa ideal), los recursos que la sociedad emplea para su tratamiento y otras acciones relacionadas, podrían ser utilizados para fines alternativos; de este modo, y en sentido inverso, los costes relevantes de la enfermedad estudiada serían aquellos que desaparecerían de desaparecer ésta ${ }^{5,19}$. En el caso del alcohol, la confrontación de la realidad con la alternativa ideal (inexistencia de consumo de alcohol) plantea asunciones discutibles, ya que considerar que la alternativa al consumo de alcohol será necesariamente el consumo de agua mineral u otros comportamientos saludables, es optimista y potencialmente irreal ${ }^{5}$. Sin embargo, el problema de la asunción de alternativas ideales no es el que más discrepancias suscita, centrándose las polémicas en qué costes deberían, o no, ser considerados y, sobre todo, en cómo deberían estos costes ser valorados. 
La racionalidad económica (teoría de la utilidad) asume que los consumidores valoran su propio consumo y que buscan racionalmente la maximización de su utilidad, aun sujetos a determinadas restricciones (como, por ejemplo, su nivel de renta). De este modo, se asume que cuando un individuo consume alcohol, el coste de la compra es compensado por los beneficios que el consumidor obtiene de la misma. Sin embargo, las conductas adictivas violan la asunción de comportamiento racional, implicando limitaciones para el análisis económico. Las aproximaciones posibles para afrontar este problema son: 1) considerar el alcohol como un producto convencional, asumiendo que incluso las personas dependientes consumen racionalmente, y tratar la transacción como cualquier otra transacción racional; 2) considerar que existe un dintel de consumo de alcohol, por encima del cual estaríamos ante un consumo abusivo ${ }^{3}$. Esto permitiría contabilizar el gasto en alcohol como costes del escenario actual frente a un escenario de consumo no abusivo, con el problema esencial de determinar cuál es el dintel que identifica un determinado nivel de consumo como abusivo.

Los estudios de coste de la enfermedad estiman el impacto de la enfermedad como una medida de bienestar social, estrechamente vinculada al Producto Interior Bruto (PIB). El PIB recoge todas las transacciones del mercado, valorándolas a su coste marginal, que puede considerarse igual al precio de mercado (incluyendo los impuestos indirectos). Por tanto, un incremento o decremento en el PIB puede considerarse como un incremento o decremento -en igual número de unidades monetarias- en la utilidad de los consumidores, pero los cambios internos -costes privados- no suponen cambios en esta utilidad. De otro modo, si los consumidores dejan de comprar agua mineral para consumir zumos, los costes privados variarán pero no se reducirá la utilidad de los consumidores. Esta situación sería similar para el alcohol, salvo que el consumo de esta sustancia va asociado a perdidas de salud, acci- dentes de tráfico y delitos que requieren el uso de recursos sanitarios y sociales, además de a perdidas productivas y otros problemas. De este modo, el consumo de alcohol produce, además de los costes que recaen exclusivamente sobre los agentes que realizan directamente el consumo (costes privados), la aparición de costes externos o externalidades ${ }^{36,37}$ que, en el contexto de los estudios de CdE suelen ser llamados costes sociales por hacer referencia a los costes soportados por el resto de la sociedad ${ }^{5}$.

En los estudios de CdE sólo los costes sociales -los que realiza la sociedad a causa de los bebedores- deben ser considerados, ya que son los únicos relevantes desde un punto de viste de las políticas públicas. Así, los tratamientos médicos soportados por el propio bebedor no formarían parte del coste de la enfermedad, sino parte del coste privado del bebedor. Nótese que "social", en este contexto, no es sinónimo de público o privado. Por ejemplo, si los seguros privados suben sus primas para compensar los costes de los accidentes causados por el alcohol, estaríamos también ante un coste social.

\section{OPERATIVA DE LOS ESTUDIOS DEL COSTE DE LA ENFERMEDAD}

La operativa de los estudios del CdE en el abuso de alcohol incluye 3 elementos $\left.{ }^{19}: 1\right)$ identificar las consecuencias del abuso de alcohol, 2) documentar la causalidad entre el abuso de alcohol y tales consecuencias y, en su caso, establecer que parte de tales sucesos sería atribuible al alcohol, y 3) asignar valores monetarios.

\section{Las consecuencias del abuso de alcohol.}

El primer paso en un estudio del $\mathrm{CdE}$, y aspecto crítico para el análisis posterior, es identificar y definir las consecuencias negativas que se consideran asociadas a la enfermedad estudiada y que permitirán definir las 
categorías de costes a considerar. Estas consecuencias deben ser tangibles y susceptibles de medición, tanto en su incidencia como en el nivel de recursos que implica su existencia. En términos generales, existe una importante coincidencia entre estudios en las grandes categorías de consecuencias que deben ser identificadas y analizadas, aunque ocasionalmente los contenidos de cada una de estas categorías no son los mismos. Estas consecuencias pueden describirse como:

\section{Tratamientos por abuso de alcohol.}

Esta categoría de costes se refiere a los servicios prestados a causa del abuso del alcohol y suele ser la que dispone de información mas fiable. La atención sanitaria suele ser codificada mediante la Clasificación Internacional de Enfermedades (CIE) que incluye diversos códigos diagnósticos relacionados directamente con el alcohol, aspecto que permite atribuir los servicios prestados por un proveedor de cuidados al abuso del alcohol. En muchas ocasiones, debido a la inexistencia de información en algunos ámbitos (atención primaria, servicios socio-sanitarios) puede ser necesario extrapolar resultados a partir de la información hospitalaria.

2. Tratamientos por enfermedades asociadas y traumatismos. El abuso de alcohol se asocia a diversas enfermedades (cirrosis, desórdenes nutricionales y metabólicos, traumatismos por accidentes de tráfico y otros, alteraciones mentales, etc.) cuya inclusión como coste requiere desarrollar estimaciones del gasto sanitario atribuible al consumo de alcohol. Usualmente se identifica en primer lugar el volumen de cada una de las enfermedades asociadas, para posteriormente aplicarles estimadores de la proporción atribuible al abuso del alcohol (fracción atribuible) obtenidas de estudios epidemiológicos. La aplicación de las fracciones atribuibles requiere evaluar cuidadosamente el rigor de la literatura revisada. Los estudios más apropiados para extraer las fracciones atribuibles son aquellos que utilizan estándares estadísticos rigurosos para identificar los diferentes factores de riesgo (entre los cuales se incluye el abuso de alcohol) y su relación de causalidad con el problema estudiado.

3. Prevención. La principal fuente de información en este capítulo será el presupuesto del gobierno. Gran parte de estos servicios se materializan en campañas publicitarias y esfuerzos formativos.

4. Mortalidad prematura. Los sistemas sanitarios consideran los datos de mortalidad como buenos indicadores del nivel de salud de la población. Generalmente los datos de mortalidad se asocian a la causa de la muerte. Es tarea fácil el acceder a este tipo de datos, aunque -como en el caso de las enfermedades asociadas- es necesario considerar las muertes no relacionadas directamente con el abuso de alcohol, pero en las cuales el consumo de alcohol actúa como factor de riesgo.

\section{Pérdidas de productividad laboral.}

Suele incluir tanto la pérdida de días de trabajo por acudir a tratamientos, como las pérdidas de productividad durante el período laboral. Los alcohólicos que reciben cuidados hospitalarios, residenciales o ambulatorios pierden días de trabajo. Este coste es fácilmente identificable.... Pero el coste por pérdidas de productividad laboral más importante no deriva de la pérdida de días de trabajo, sino de la disminución de productividad durante la realización de la actividad productiva. Numerosos trabajos demuestran que los individuos que abusan de sustancias son menos productivos en el trabajo que trabajadores no - abusadores con el mismo grado de experiencia y capacidad. Aquí el trabajo de estimación partirá de los estimadores de coste identificados en estos estudios.

\section{Destrucción de la propiedad por crí-} menes o accidentes.- Como para el resto de componentes de coste, existen dos tipos de datos que nos serán necesarios para calcular este coste: por un lado, los datos relativos a la incidencia y el coste de estos eventos, y después las estimaciones de la fracción atribuible de dichos costes al abuso del alcohol.

7. Gastos de justicia penal.- Como para el gasto sanitario, existen gastos directamente 
relacionados con el abuso de alcohol (incluso por definición) mientras que otros los están de una forma indirecta. Se identifican básicamente tres tipos de coste: actividades de justicia penal dedicadas exclusivamente a combatir las consecuencias del abuso del alcohol, actividades de entidades de justicia criminal referidas al uso ilegal de sustancias y actividades referidas a crímenes que se creen causados por el abuso de alcohol. Los datos necesarios y las estimaciones pertinentes se pueden extraer de las bases de datos de las autoridades legales, tribunales de justicia y correccionales.

\section{Pérdidas laborales de las víctimas.-}

También existe un coste, relativamente reducido, derivado de las pérdidas de días de trabajo de las víctimas de crímenes o sucesos penales derivados del abuso del alcohol. La estimación de dicho coste supone tener información acerca del número de crímenes por año, del tiempo productivo perdido a causa de estos crímenes, y de la proporción de varios tipos de crímenes atribuidos al abuso del alcohol.

9. Encarcelamiento.- Cuando los individuos son encarcelados son apartados de la economía productiva. Esto constituye una pérdida de productividad potencial para la economía. Los datos acerca de las poblaciones encarceladas ( y el coste que generan) son generalmente fácilmente obtenibles.

10. Costes de la carrera criminal.- Esta es probablemente la categoría de coste más esotérica de las incluidas en este estudio. Se entiende por coste de la carrera criminal aquel que significa una pérdida de productividad derivada de la participación en la producción o el comercio de sustancias ilegales. Los estimadores obtenibles tendrían escasa o nula significación estadística, puesto que se basarían en el juicio de expertos.

\section{Causalidad y fracciones atribuibles.}

El establecimiento de causalidad y la determinación de las fracciones atribuibles es, probablemente, el aspecto más crítico de los estudios del CdE. En algunos casos (diagnósticos de problemas alcohólicos de la CIE) la atribución de determinadas consecuencias al abuso de alcohol no plantea problemas. Sin embargo, la situación usual es que el abuso de alcohol sea sólo una de las múltiples causas de una determinada consecuencia (enfermedades asociadas, accidentes, criminalidad, perdidas de productividad), siendo necesario determinar en que medida tales consecuencias pueden ser atribuidas al alcohol. Este problema es más complejo si se tiene en cuenta la distinción entre asociación y causalidad (el hecho de que el conductor implicado en un accidente haya bebido no quiere decir que el alcohol haya sido la causa del accidente), aspecto que obliga a estimar el riesgo añadido de los bebedores respecto a los no bebedores para cada uno de las situaciones en las que el alcohol es una causa contribuyente, incluyendo la criminalidad y las perdidas de productividad.

Afortunadamente existen numerosos estudios epidemiológicos que han establecido el riesgo relativo de padecer desórdenes concretos en función de diferentes niveles de consumo de alcohol y permiten establecer las fracciones atribuibles de morbilidad y mortalidad relacionadas con el alcohol con un alto grado de fiabilidad (ver tabla 2). No obstante, muchos de estos estudios se han desarrollado sobre poblaciones de pacientes hospitalizados o en tratamiento, y es dudoso que los riesgos de mortalidad y morbilidad en la población general de consumidores de alcohol sean los mismos que en las poblaciones de pacientes tratados. Igualmente, las fracciones atribuibles varían entre sociedades, entre grupos sociales y en el tiempo.

\section{Modelos de estimación de costes.}

El último paso en un estudio del CdE es asignar valores monetarios a las consecuencias del abuso de alcohol. Buena parte de esta asignación puede realizarse utilizando los precios de mercado, tanto directamente si se trata de recursos para los que existe un mercado (primas de seguros), como utilizando 
Tabla 2. Listado de códigos diagnósticos y fracciones atribuibles de morbilidad relacionada con el alcohol.

\begin{tabular}{|c|c|c|}
\hline Categorías de enfermedad & Códigos ICD-9 & Fracciones atribuibles (FA) \\
\hline $\begin{array}{l}\text { Enfermedades infecciosas } \\
\text { Tuberculosis } \\
\text { Neumonía, gripe }\end{array}$ & $\begin{array}{c}011-013,017,018 \\
480-487\end{array}$ & $\begin{array}{c}25 \\
5\end{array}$ \\
\hline $\begin{array}{l}\text { Neoplasiasmalignas } \\
\text { Labio, boca, faringe } \\
\text { Esófago } \\
\text { Estómago } \\
\text { Hígado, vías biliares } \\
\text { Laringe } \\
\text { Mama }\end{array}$ & $\begin{array}{c}140-149,230 \\
150,230.1 \\
151 \\
155,230.8 \\
161,231.0 \\
174,233.0\end{array}$ & $\begin{array}{l}50 \\
80 \\
20 \\
29 \\
50 \\
13\end{array}$ \\
\hline $\begin{array}{l}\text { Enfermedades cardiovasculares } \\
\text { Cardiomiopatía } \\
\text { Hipertensión arterial } \\
\text { Accidente vascular cerebral }\end{array}$ & $\begin{array}{c}425.1,425.4-425.9 \\
401-405,642.0 \\
642.2,642.9 \\
430-438\end{array}$ & $\begin{array}{l}40 \\
11 \\
7\end{array}$ \\
\hline $\begin{array}{l}\text { Enfermedades digestivas } \\
\text { Esófago, estómago, duodeno } \\
\text { Otras cirrosis, hepatopatías } \\
\text { Pancreatitisaguda } \\
\text { Pancreatitiscróncia }\end{array}$ & $\begin{array}{c}530-537 \\
571.5,571.6 \\
577.0 \\
577.1\end{array}$ & $\begin{array}{l}10 \\
74 \\
47 \\
72\end{array}$ \\
\hline $\begin{array}{l}\text { Otras } \\
\text { Quemados } \\
\text { Demencia } \\
\text { Epilepsia } \\
\text { Convulsiones } \\
\text { Traumatismos } \\
\text { (inlcuye accidentes de tráfico) }\end{array}$ & $\begin{array}{c}940 \\
290.1-4,294 \\
345.1,345.3,345.9 \\
780.3 \\
800.0,909.9,921.0- \\
939.9,950.0-959.9\end{array}$ & $\begin{array}{l}25 \\
11 \\
30 \\
41 \\
40\end{array}$ \\
\hline $\begin{array}{l}\text { Enfermedades totalmente atribuibles } \\
\text { al alcohol } \\
\text { Psicosis alcohólica } \\
\text { Síndrome de dependencia } \\
\text { Abuso de alcohol } \\
\text { Cardiomiopatía alcohólica } \\
\text { Gastritis alcohólica } \\
\text { Hígado graso } \\
\text { Hepatitis alcohólica aguda } \\
\text { Cirrosis alcohólica } \\
\text { Daño hepático alcohólico no especificado } \\
\text { Intoxicación alcohólica aguda } \\
\text { Efecto tóxico del alcohol } \\
\text { Polineuropatía alcohólica } \\
\text { Daño fetal por alcohol } \\
\text { Síndrome alcohólico fetal } \\
\text { Nivel elevado de alcohol en sangre } \\
\text { Alcoholismo } \\
\text { Alcoholismo en la familia } \\
\text { Pruebas de alcoholemia } \\
\text { Gribaje especial por alcoholismo }\end{array}$ & $\begin{array}{c}291 \\
303 \\
305.0 \\
425.5 \\
535-3 \\
571.0 \\
571.1 \\
571.2 \\
571.3 \\
\text { E860.1 } \\
980 \\
357.5 \\
655.4 \\
760.71 \\
790.3 \\
\text { V11.3 } \\
\text { V61.41 } \\
\text { V70.4 } \\
\text { V79.1 }\end{array}$ & $\begin{array}{l}100 \\
100 \\
100 \\
100 \\
100 \\
100 \\
100 \\
100 \\
100 \\
100 \\
100 \\
100 \\
100 \\
100 \\
100 \\
100 \\
100 \\
100 \\
100\end{array}$ \\
\hline
\end{tabular}


costes si se trata de recursos del sector público (costes de los servicios sanitarios, policiales, judiciales,...). En este último caso existen diversas limitaciones, pero en generar el uso de costes medios o fracciones atribuibles del presupuesto global de un determinado servicio son aproximaciones aceptables.

Los costes indirectos de perdidas de producción suelen valorarse utilizado el salario medio interprofesional y pueden incluir el "salario" de las amas de casa. Una estimación rigurosa de estos costes -crítica, ya que pueden constituir la parte más voluminosa del coste de la enfermedad- debería incluir diversos ajustes en función de los niveles de empleo, edad y sexo (el consumo de alcohol no se distribuye uniformemente, estando mas afectados los adultos -varones- jóvenes)

Existen dos aproximaciones clásicas para estimar los costes económicos debido al consumo de sustancias: la aproximación del "capital humano" y la aproximación "demográfica". La diferencia fundamental entre ambas es la manera en que tratan los costes de la mortalidad prematura. En la aproximación del capital humano, la pérdida de producción derivada de la muerte de un trabajador se calcula a partir de los ingresos presentes, aplicando una tasa de descuento para estimar las ganancias futuras. La aproximación demográfica compara el nivel y la estructura poblacional actual con la de un escenario de población "sana" - exenta de muertes por consumo de alcohol. Estas dos aproximaciones son complementarias, antes que contradictorias. La aproximación demográfica plantea el siguiente supuesto: "Suponga que nunca se ha consumido alcohol y que no han existido problemas relacionados con su consumo"; el método del capital humano pregunta: "Suponga que el consumo de alcohol y los problemas relacionados con este acabasen hoy".

La aproximación del capital humano estima los costes presentes y futuros debidos a mortalidad relacionada con el consumo de alcohol en el presente año, mientras que la aproximación demográfica mide el coste actual de la mortalidad relacionada con el abuso de alcohol en el pasado y el presente. Cabe esperar que los resultados que se obtengan con una u otra aproximación difieran siempre, ya que responden a preguntas diferentes. Es por lo tanto imprescindible explicitar cuál de los dos métodos se ha utilizado. Los estudios del coste de la enfermedad deberían idealmente utilizar ambos enfoques, y comparar los resultados.

\section{ESTUDIOS DEL COSTE DEL ALCOHOLIS- MO EN ESPAÑA}

No ha habido una gran proliferación de estudios sobre el coste del alcoholismo en España. El tema del alcohol ha suscitado reflexiones acertadas acerca de la importancia de las externalidades financieras generadas por su consumo ${ }^{32}$.

En este apartado nos vamos a centrar en un trabajo de Portella de reciente publicación ${ }^{34}$. El objetivo de dicho estudio fue evaluar el impacto económico en términos de costes sanitarios del abuso del alcohol en España. Se utilizaron fuentes de información secundarias, siempre las más recientes disponibles, utilizando diferentes métodos operativos para el cálculo de cada tipología de coste (ver tabla 3). El período considerado para el cálculo de los costes fue el de un año natural, actualizando los precios a pesetas constantes para 1996. El enfoque se basó en la prevalencia de casos por período. Toda la información se ha refirió (o se extrapoló a partir de datos a nivel de Comunidad Autónoma) al conjunto de España.

Los resultados del estudio cifran en 637.718 millones de pesetas los costes sanitarios sociales anuales ocasionados en España por el consumo excesivo de alcohol. En términos comparativos, esta cifra representó el 16\% del presupuesto sanitario de las Administraciones Públicas para el año 1997. Los costes considerados se agruparon taxonómicamente en costes directos (directamente imputables al consumo excesivo de alcohol) e indirectos (los relacionados con la repercusión de los 
Tabla 3. Tipologías de coste consideradas y métodos de estimación

\begin{tabular}{ll}
\hline Concepto & Método de cálculo \\
\hline $\begin{array}{l}\text { Visitas } \\
\text { ambulatorias }\end{array}$ & $\begin{array}{l}\text { Aplicación de las fracciones atribuibles de morbilidad al total de visitas } \\
\text { realizadas por la Especialidad de Medicina General para cada código } \\
\text { diagnóstico ICD-9 relacionado con el consumo de alcohol para obtener } \\
\text { el número de consultas, y multiplicación por el coste unitario de una } \\
\text { visita en Atención Primaria. }\end{array}$
\end{tabular}

\begin{tabular}{ll}
\hline $\begin{array}{l}\text { Centros } \\
\text { especiales }\end{array}$ & $\begin{array}{l}\text { Estimación del número de pacientes que someten en un año a trata- } \\
\text { mientos en centros específicos y multiplicación por el coste medio por } \\
\text { paciente en un centro especial. }\end{array}$ \\
\hline
\end{tabular}

Ingresos Aplicación de fracciones atribuibles de morbilidad, extrayendo el núhospitalarios mero total de enfermos dados de alta durante un año para un ICD-9 relacionado con el consumo de alcohol, y mulitplicación el cálculo del gasto corriente medio por alta.

\begin{tabular}{ll}
\hline $\begin{array}{l}\text { Urgencias } \\
\text { hospitalarias }\end{array}$ & $\begin{array}{l}\text { Cálculo del porcentaje de urgencias hospitalarias atribuido al alcohol y } \\
\text { multiplicación por el coste medio por consulta de urgencia. }\end{array}$ \\
\hline $\begin{array}{l}\text { Otros gastos } \\
\text { sanitarios }\end{array}$ & $\begin{array}{l}\text { Cálculo de la fracción de siniestralidad laboral atribuible al consumo } \\
\text { excesivo de alcohol y multiplicación por el coste medio sanitario de } \\
\text { un accidente laboral con baja. }\end{array}$ \\
\hline
\end{tabular}

$\begin{array}{ll}\text { Pérdidas } & \text { Aplicación de la fracción atribuible del alcohol en accidentes de tráfico } \\ \text { materiales } & \text { y mulitplicación por el coste medio de las pérdidas materiales por } \\ & \text { siniestro. }\end{array}$

\begin{tabular}{ll}
\hline $\begin{array}{l}\text { Recursos } \\
\text { adicionales }\end{array}$ & $\begin{array}{l}\text { Aplicación de la fracción atribuible al alcohol en la utilización de servi- } \\
\text { cios judiciales y penales a los costes procedentes de las partidas de } \\
\text { gasto correspondientes dentro de los Presupuestos del Estado. }\end{array}$ \\
\hline
\end{tabular}

Subvenciones y Partidas presupuestarias de programas destinados total o en parte a ayudas combatir los problemas sociales derivados del consumo de alcohol.

\begin{tabular}{ll}
$\begin{array}{l}\text { Absentismo } \\
\text { laboral }\end{array}$ & $\begin{array}{l}\text { Producto del coste unitario de un día de baja por el número de días } \\
\text { perdidos a causa del consumo de alcohol, por la fracción de la preva- } \\
\text { lencia en el mundo laboral respecto a la población activa ocupada. }\end{array}$ \\
\hline $\begin{array}{l}\text { Reducción } \\
\text { eficiencia }\end{array}$ & $\begin{array}{l}\text { Aplicación de la tasa de prevalencia del alcoholismo en el mundo labo- } \\
\text { ral, multiplicada por el salario promedio por trabajador. }\end{array}$
\end{tabular}

problemas relacionados con el alcohol sobre el sistema productivo). En la tabla 3 se detallan los costes considerados, así cómo el coste total atribuido a cada tipo de coste y su importancia sobre el total global.

El estudio no incluyó el coste de las consultas externas en centros hospitalarios, las indemnizaciones por muerte e invalidez de accidentes, los costes del síndrome alcohólico fetal, el coste de los años potenciales de vida productiva perdidos y el coste monetario de los años potenciales de vida perdidos. Al haber calculado unas estimaciones que no incluyen el total de costes derivados del abuso del alcohol, los autores concluyen que sí son como mínimo los que se producen en nuestro país. 
Tabla 4. Impacto económico anual del consumo de alcohol en España.

\begin{tabular}{|c|c|c|}
\hline Conceptos & Coste total (millones de pesetas) & Porcentaje (\%) \\
\hline Directos & 228.429 & $35,8 \%$ \\
\hline Visitas ambulatorias & 34.599 & 5,4 \\
\hline Centros hospitalarios & 18.028 & 2,8 \\
\hline Ingresos hospitalarios & 93.664 & 14,7 \\
\hline Urgencias hospitalarias & 10.481 & 1,6 \\
\hline Otros gastos sanitarios & 20.329 & 3,2 \\
\hline Pérdidas materiales & 1.675 & 0,3 \\
\hline Recursos adicionales & 48.956 & 7,7 \\
\hline Subvenciones ayudas & 714 & 0,1 \\
\hline Indirectos & 409.288 & 64,2 \\
\hline Absentismo laboral & 121.219 & 19,0 \\
\hline Reducción eficiencia & 288.069 & 45,2 \\
\hline Total & 637.718 & $100 \%$ \\
\hline
\end{tabular}

\section{LIMITACIONES DE LOS ESTUDIOS DEL COSTE DE LA ENFERMEDAD}

Los estudios del CdE alcohólica han recibido diversas críticas ${ }^{12-19,37,38-42}$ en relación con la identificación, medida y cuantificación de los costes, así como con su propia utilidad.

Respecto a la identificación de los costes, las críticas se han centrado en ${ }^{19}$ :

- Aunque la idea básica es que sólo los costes sociales son relevantes desde un punto de vista de los fundamentos económicos (y de las políticas públicas), existe cierta tendencia a considerar que en sustancias adictivas como el alcohol las preferencias individuales tiene menos importancia, con su correlato de confusión entre costes sociales (externalidades) y costes privados. Por ejemplo, se ha señalado ${ }^{15}$ que de los costes del alcoholismo en Estados Unidos calculados por Rice $^{3}$ sólo 10 billones de dólares eran costes externos, mientras que 60 billones eran costes privados.

- Los seguros sociales tienen una función redistributiva (desde quienes pagan impuestos a los beneficiarios) pero no reducen la disponibilidad global de recursos y, por tanto, su inclusión como costes sociales -frecuente en los estudios CdCes inadecuada.

- En una economía con pleno empleo las pérdidas de productividad reducirían el PIB, pero si las tasas de desempleo son elevadas las pérdidas sociales se reducirían al coste del reemplazamiento del trabajador alcohólico, ya que este podría ser sustituido por otro no bebedor. En estas circunstancias sólo estos últimos costes -y no las pérdidas de productividad globales- deberían considerarse costes sociales.

- En un mundo racional, con consumidores completamente informados y no adictos, las decisiones individuales de consumo se tomarían según los costes y beneficios y marginales del alcohol para cada individuo. Estos costes y beneficios son privados y, por tanto, no son costes sociales. Pero si debido a la falta de información o la propia adicción no pueden realizarse elecciones racionales (por ejemplo, la adicción podría definirse como un consumo que no reporta beneficios) una parte de estos costes privados son perdidas de bienestar y, por tanto, costes sociales ${ }^{3,40}$.

- Es conocido que, aunque una minoría de grandes consumidores tiene un elevado riesgo de problemas de salud, es una 
mayoría de consumidores de menor riesgo la que produce el mayor número de casos, y esta situación plantea el problema de si deberían considerarse como costes sociales sólo los de los grandes bebedores (asumiendo que los moderados serían consumidores informados).

Respecto a la medición, es obvio que los costes varían según grupos sociales y que su uso requiere la existencia de buena información acerca de los patrones de consumo de la población, especialmente porque los problemas de salud dependen -en mayor o menor medida- de muchos otros aspectos (cuidado, alimentación, etc.) de la vida de los bebedores. En la práctica los problemas incluyen tanto la subestimación de algunos parámetros (por ejemplo, si los censos y encuestas excluyen -o pierden por falta de respuesta a los grandes bebedores), como la sobreestimación de otros (por ejemplo, atribuyendo al alcohol riesgos del tabaco $u$ otros estilos de vida no saludables que pueden ser mas frecuentes en bebedores). Esta situación, junto a la combinación de fuentes para la obtención de fracciones atribuibles y la extrapolación de los riesgos relativos de unos entornos a otros, produce un alto grado de incertidumbre sobre las mediciones de costes.

Igualmente, las pérdidas potenciales de productividad derivadas del consumo de alcohol plantean problemas especialmente importantes a causa de la dificultad que conlleva calcular el valor de los bienes y servicios que no se producirán a causa del consumo de alcohol. Asumiendo que los ingresos de un individuo corresponden al valor de lo que produce, los costes de la pérdida de productividad se pueden medir a partir de la reducción de los ingresos experimentada por individuos con desórdenes relacionados con el alcohol. Por ejemplo, una estimación estadounidense de 1985 halló que los individuos que cumplieron los criterios clínicos de abuso de alcohol o de dependencia alguna vez en el tiempo sufrieron una disminución de sus ingresos de entre 1,5 al $18,7 \%$, en función de la edad y el sexo, comparado con aquellos que no cumplieron el diagnóstico"1.
Respecto a la cuantificación de los costes, existe cierto consenso en utilizar los precios de mercado para valorar los directos (servicios sanitarios, ausencias laborales,...), pero las aproximaciones pueden ser muy diversas cuando se trata de calcular el valor de la vida humana, y los estudios muestran que los diversos métodos posibles (valores implícitos, capital humano, ...) produce estimaciones muy diferentes. Esto mismo sucedería con los costes intangibles (dolor, sufrimiento,...). Además de los citados problemas de medición y estimación, las estimaciones de los costes económicos totales basadas en los estudios de coste de la enfermedad tienen varias limitaciones.

Ciertas consecuencias se suelen omitir en los estudios del coste de la enfermedad. Entre estas estarían los costes del dolor y del sufrimiento entre los consumidores, pero también sus familias, amigos y colaboradores. También los efectos del abuso del alcohol sobre la estabilidad familiar (e incluso los efectos secundarios del abuso sobre otros mercados como los seguros, automóviles y bebidas sin alcohol). El tratamiento y la medición de los costes intangibles plantea importantes inconvenientes, en la medida en que la reducción de costes intangibles no se traduce en la liberación de recursos para usos alternativos (por ejemplo, una reducción del dolor, aún siendo un importante beneficio, no permite transferir directamente este beneficio a otras personas) ya que no existe un mercado de los beneficios derivados de la reducción de costes intangibles. Es por lo tanto extremamente difícil dar un valor a los costes intangibles por lo que generalmente, se excluyen estos costes.

Respecto a la utilidad de los estudios CdC, la alternativa de comparación, en la que no existe abuso de alcohol, es una hipótesis que conlleva diversos problemas: 1) no es alcanzable bajo ningún supuesto ya que existe una parte del coste no recuperable debida a costes soportados en la actualidad debido al abuso del alcohol en el pasado; 2) no es obvio que un escenario sin alcohol sea una alternativa más apropiada desde el punto de vista de la sociedad que un escenario con un consu- 
mo moderado de alcohol, entre otras cosas porque la relación entre riesgo y consumo no es lineal y el consumo moderado tiende a mostrar un menor riesgo que el consumo elevado, pero también que la abstinencia completa; 3$)$ la estimación de costes derivada de los estudios CdE incluye tanto costes evitables como no evitables, lo que implica que las estimaciones no pueden interpretarse como el beneficio potencial para la sociedad; 4) las estimaciones del coste total del alcohol sólo valoran costes -y no beneficios- por lo que son de escasa utilidad para evaluar medidas políticas o acciones preventivas.

\section{CONCLUSIONES}

Los estudios del coste del alcoholismo han proliferado en los últimos años, con desarrollo de interés incluso en España, al tiempo que han venido desarrollándose su marco teórico y estandarizándose los aspectos metodológicos. Pese a diversas limitaciones, las estimaciones del coste económico total del abuso de alcohol nos aproximan a la magnitud del problema, lo que es importante a la hora de dirigir la atención de los decisores y de atraer recursos hacia actividades orientadas a reducir dicho coste. Estas estimaciones también ayudan a identificar cuales son los aspectos del problema que suponen un coste más elevado, y por lo tanto permitirían -hasta cierto punto- orientar la dirección de las políticas. La calidad de estos estudios, y por tanto su valor real, va a depender de la pulcritud en la identificación, medida y cuantificación de los costes. En este terreno, el ajuste a las guías y la explicitación detallada de las fuentes y métodos utilizados es un requisito necesario para su credibilidad.

\section{BIBLIOGRAFÍA}

(1) Rice DP. Estimating the Cost of Illness. DHEW Publication (PHS) 947-6. Rockville, MD: U.S. Department of Health, Education and Welfare, 1966.
(2) Maynard A, Hardman G, Whelan A. Measuring the social costs of addictive substances. $\mathrm{Br} J$ Addiction 1987; 82:701-706.

(3) Collins DJ, Lapsley HM. Estimating the Economic Costs of Drug Abuse in Australia. National Campaign Against Drug Abuse. Monograph Series 15. Canberra: Australian Government Printing Service, 1991.

(4) Single E, Collins D, Easton B, Harwood H, Lapsley H, Maynard A. International Guidelines for estimating the costs of substance abuse. Ottawa: Canadian Centre on Substance Abuse, 1996.

(5) Harwood H, Fountain D, Livermore G. The economic costs of alcohol and drug abuse in the United States, 1992. NIH Publication 984327. Bethesda, Ma: National Institutes of Health, 1998.

(6) Robson L, Single E. Literature Review of Studies on the Economic Costs of Substance Abuse. Ottawa: Canadian Centre on Substance Abuse, 1995.

(7) Hodgson TA, Meiners M.Guidelines for Cost of Illness Studies in the Public Health Service. Task Force on Cost of Illness Studies. Washington: U.S. Public Health Service, 1979.

(8) Hodgson TA, Meiners M. Cost-of-Illness Methodology: A Guide to Current Practices and Procedures. Milbank Memorial Fund Quarterly 1982; 60:429-462.

(9) Harwood H, Napolitano DM, Christensen PL, Collins JJ. Economic Costs to Society of Alcohol and Drug Abuse and Mental IIIness: 1980. Research Triangle Park, NC: Research Triangle Institute, 1984

(10) Rice DP, Hodson TA, Kopstein AN. The economic costs of illness: a replication and update. Health Care Financing Rev 1985; 7:61-80.

(11) Rice DP, Kelman S, Miller LS, Dunmeyer S. The Economic Cost of Alcohol and Drug Abuse and Mental Illness 1985. DHHS Publication 901694. San Francisco: Institute for Health and Aging, University of California, 1990.

(12) DiNardo J. A Critical Review of the Estimates of the "Costs" of Alcohol and Drug Use. Los Angeles, CA: University of California - RAND Corporation, 1992.

(13) Drummond M. Cost-of-illness studies: a major headache? Pharmacoeconomics 1992; 2:1-4

(14) Heien DM, Pittman DJ. The economic costs of alcohol abuse: an assessment of current 
methods and estimates. J Studies Alcohol 1989; 50:567-579.

(15) Heien DM, Pittman DJ. The external costs of alcohol abuse. J Studies Alcohol 1993; 54: 302-7.

(16) Estimating costs associated with alcohol abuse: towards a pattern approach. International Center for Alcohol Policies. August 1999.

(17) Koopmanschap MA. Cost-of-illness studies: useful for health policy? Pharmaco-Economics 1998; 14: 143-148.

(18) Wiseman V, Mooney G. Burden of illness estimates for priority setting: a debate revisited. Health Policy 1998; 43: 243-251.

(19) Maynard A, Godfrey C, Hardman G. Conceptual issues in estimating the social costs of alcohol. Ottawa: Canadian Centre on Substance Abuse, 1994.

(20) Reynolds DJ. The Cost of Road Accidents. J Royal Stat Soc 1956; 119:393-408.

(21) Fein R. Economics of Mental Illness. New York: Basic Books, 1958.

(22) Pritchard HM. Economic Costs of Abuse of and Dependency on Alcohol in Australia. In: Kiloh LG, Bell DS, eds. Proceedings of the 29th International Congress on Alcoholism and Drug Dependence, February 1970. Sydney, Australia: Butterworths, 1971.

(23) Berry R, Boland J. The Economic Costs of Alcohol Abuse - 1972. Brookline, MA: Policy Analysis Inc., 1973.

(24) A.D. Little, Inc. Social Costs of Drug Abuse. Report to the Special Action Office of Drug Abuse Policy. Washington: the White House, 1973.

(25) Collins D, Lapsley H. The Social Costs of Drug Abuse in Australia in 1988 and 1992. Camberra: Commonwealth Department of Human Services and Health. Australian Government Publishing Service, 1996

(26) Single E, Robson L, Xie X, Rehm J. The Costs of Substance Abuse in Canada. Ottawa, Canada: Canadian Centre on Substance Abuse, 1996.

(27) Single E, Robson L, Xie X, Rehm J. The economic costs of alcohol, tobacco and illicit drugs in Canada, 1992. Addiction 1998;93:991-1006.

(28) Xie X, Rehm J, Single E, Robson L, Paul J. The economic costs of alcohol abuse in Ontario.Pharmacol Res 1998;37:241-249.

(29) Devlin NJ, Scuffham PA, Bunt LJ. The social costs of alcohol abuse in New Zealand.
Addiction 1997:92:1491-1505.

(30) Nakamura K, Tanaka A, Takano T. The social cost of alcohol abuse in Japan. J Studies Alcohol 1993; 54:618-625.

(31) Rovira J, Oriols P. Aproximació al cost social de I'alcoholisme a Catalunya. Barcelona: Departament de Sanitat i Seguretat Social. Generalitat de Catalunya, 1982.

(32) González López-Valcárcel B Equidad, superequidad y externalidades financieras: los casos del tabaco y del alcohol. Madrid: Ministerio de Sanidad y Consumo, Asociación de Economía de la salud, 2000.

(33) Aproximación al estudio de los costes sociales del alcoholismo en la Comunidad Autónoma Vasca Bilbao: Departamento de Estudios y Planificación de la Cámara de Comercio y Navegación de Bilbao.

(34) Portella E, Carrillo E, Ridao M, Ribas E, Ribó C, Salvat $\mathrm{M}$ El alcohol y su abuso: impacto socioeconómico. Madrid: Panamericana, 1998.

(35) Portella E, Ridao M, Salvat C, Carrillo C. Costes sanitarios del alcoholismo. Aten Primaria 1998; 22: 279-84.

(36) Cook PJ, Moore MJ. Alcohol. En: Newhouse PJ, Culyer J, eds. Handbook of health economics, 2000.

(37) Melberg HO. The concepts of "cost to society" and "social cost". Oslo: National Institute for Alcohol and Drug Research, 2000.

(38) Wagstaff A. Government prevention policy and the relevance of cost estimates. Br J Addiction 1987; 82: 461-467.

(39) Shiell A, Gerard K and Donaldson C. Cost of illness studies: an aid to decision making? Health Policy 1987; 8: 317-323.

(40) Hodgson TA. Cost of illness studies: no aid to decision-making? Comments of the second opinion of Sheill et al. Health Policy 1989; 11: 57-60.

(41) Godfrey C, Hardman G. Changing the Social Cost of Alcohol. York: Centre for Health Economics, University of York, 1994.

(42) Smith K, Wright K. Informal care and economical appraisal: a discussion of possible methodological approaches. Health Economics 1994; 3: 137-8.

(43) Cook PJ. The social costs of drinking. En: The Negative Social Consequences of Alcohol Use. Oslo: Norwegian Ministry of Health and Social Affairs, 1991. 